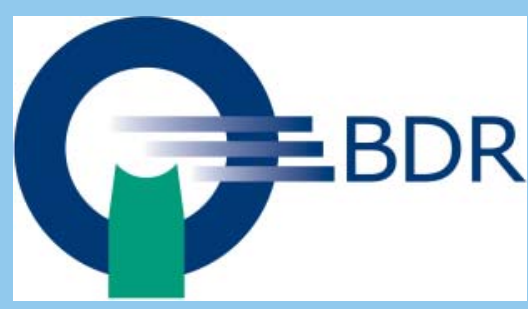

\title{
Radiologie-Update 2018
}
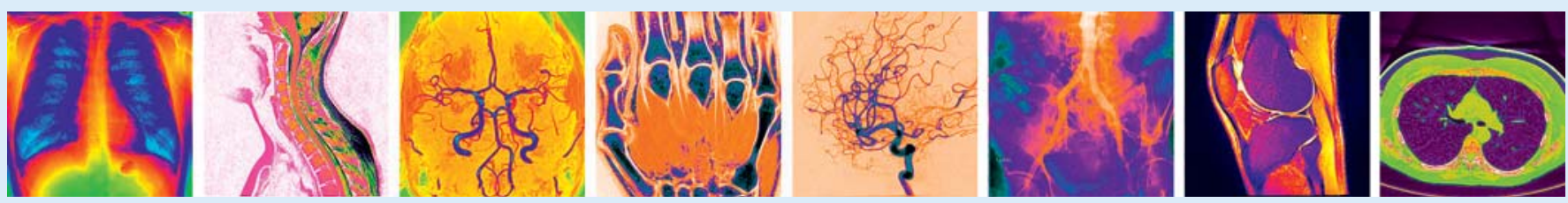

Das diesjährige Radiologie-Update in Ulm Mitte März wurde wiederum sowohl von in der Praxis wie in der Klinik tätigen Kolleginnen und Kollegen sehr gut angenommen. Die neu geschaffene eigene Kongresshomepage www.radiologie-update-ulm.de eröffnet die Möglichkeit, zurückliegende Kongresse wie auch zukünftige Veranstaltungen optimal nach- wie vorzubereiten. 2018 wird erstmalig ein eBook vorliegen, das in moderner Form ein optimales Nachlesen des Kongresses ermöglicht.

Eröffnet wurde die Veranstaltung von 2 neuroradiologischen Vorträgen. Prof. Schmitz aus Ulm stellte im Bereich Wirbelsäule neue Ansätze zur Dosisreduktion bei CT-Untersuchungen, CT und MRT-Zeichen zervikaler Traumata sowie Gefäßvarianten der Wirbelsäule vor. Für das ZNS wurden u. a. die Weiterentwicklung der 2016 vorgestellten WHO-Klassifikation der Hirntumoren, Fallsammlungen zur Klassifikation von Tumorgradingsmustern und Neuansätze zum Verständnis der hämatologischen Tumorausbreitung vorgestellt. Prof. Pham aus Würzburg fasste anschließend Neuerungen zur Schlaganfalldiagnostik wie -therapie zusammen. Hinsichtlich der Akuttherapien haben sich signifikante Neuerungen ergeben; es verschiebt sich die Indikation der interventionellen Behandlung sowohl hinsichtlich der Infarktausdehnung als auch des Infarktzeitpunkts. Mögliche pitfalls in der Infarkteinschätzung sowohl bei CT- wie MRT-Diagnostik wurden eingehend und anschaulich vorgestellt und im Anschluss lebhaft diskutiert.
Hinsichtlich der Leberdiagnostik stellte Prof. Seidensticker von der LMU München Neuerungen hinsichtlich der multiparametrischen Leber-MRT dar. Die verbesserte prätherapeutische Bildgebung erlaubt für das hepatozelluläre Karzinom nun activesurveillance-Ansätze. Stärken wie Limitation der MR-Bildgebung der Leber sowie Tipps und Tricks wurden detailliert dargestellt. Prof. Juchems aus Konstanz fasste die aktuelle Studienlage für Pankreas und Gastrointestinaltrakt zusammen. Dabei fokussierte er zum einen auf die neuroendokrinen Tumoren und duktalen Adenokarzinome des Pankreas sowie für den Gl-Trakt auf das Rektumkarzinom und Erkrankungen des Dünndarms. Die Kriterien zur Resektabilität des Pankreaskarzinoms wurden 2017 neu gefasst, ebenso die Vorgaben zum postoperativen Imaging. Hinsichtlich neuroendokriner Tumoren haben PET-CTUntersuchungen eine zunehmende Bedeutung. Split-Bolus-Protokolle stellen bei mesenterialer Ischämie einen interessanten Ansatz dar. Bei Rektumkarzinomen könnte die chemical-shift-Bildgebung einen vielversprechenden Ansatz darstellen.

Die Neuerungen der kardiovaskulären CTund MRT-Diagnostik stellte Professor Sandstede aus Hamburg zusammen. In der Primärdiagnostik der KHK erlaubt die CCTA durch die Detektion von Hochrisikoplaques eine Abschätzung des Risikoprofils. Zudem sind unter Therapie Veränderungen der Plaques mittels CT detektierbar. Die ergänzende Analyse mittels CT-Perfusion hat Potential zu einer erweiterten Prognoseabschätzung. Die MRT besticht durch ihre
Möglichkeit der Fibrosedarstellung; sie wird auch bei Patienten mit Herzschrittmacher immer häufiger angewandt und erlaubt mittels feature-tracking eine noch exaktere und quantitative Abschätzung der Herzfunktionen. Moderne CT- und MR-Angiografietechniken stellte Professor Richter aus Stuttgart hinsichtlich ihres Potentials zu einer dosisoptimierten und ggf. sogar kontrastmittelfreien Gefäßdarstellung vor. Der 3D-Druck ermöglicht bereits präinterventionell eine exakte Abschätzung der Therapiemöglichkeiten und erlaubt ein individuelles Anpassen entsprechender Gefäßprothesen.

Rechtsanwalt Markus Henkel vom Berufsverband der Deutschen Radiologen stellte in seinem Vortrag Kernpunkte des umfassenden Strukturwandels in der Radiologie dar. Im ambulanten Bereich ist die Zahl der angestellten Ärztinnen und Ärzte in den letzten Jahren deutlich angestiegen. Dies hat unterschiedliche Gründe. Unterschiedlichste Praxismodelle haben sich in der Vergangenheit entwickelt; das Modell der MVZ ist insbesondere durch überregionale Verbünde von besonderer Bedeutung. Der Trend zu überregionalen Großpraxen ist unverändert. Es zeigt sich auch ein zunehmendes Interesse des Kapitalmarkts, bedingt durch gesetzliche Vorgaben. Welche langfristigen Perspektiven sich daraus ergeben ist derzeit offen. Es gibt unterschiedliche Gründe für die Übertragung von Praxen und Praxisgemeinschaften an Investoren. Der Berufsverband beteiligt sich aktiv hinsichtlich der rechtlichen Ausgestaltung 
um Rechtsnormen zu gewährleisten, die das Patientenwohl primär im Auge haben.

Im Highlight-Vortrag lenkte Professor Forsting aus Essen die Blickrichtung auf die digitale IT-Transformation der Radiologie. Die zunehmende Mächtigkeit der artifiziellen Intelligenz und des machine-learnings werden viele Bereiche gerade in der Diagnostik fundamental umwälzen. Angesichts des signifikant gestiegenen Arbeitsumfangs für Radiologen können jedoch diese IT-Lösungen durchaus als unterstützend aufgefasst werden. Inwieweit sich das Berufsbild der Radiologie durch diese IT-Möglichkeiten wandeln wird, hängt letztlich auch von der Annahme und Transformation durch die Radiologinnen und Radiologen selbst ab.

Am zweiten Tag des Radiologie-Updates fasste Professor Uder aus Erlangen die aktuelle Studienlage der urogenitalen Radiologie zusammen. Insbesondere für PIRADSIII-Befunde ergeben sich Neuerungen für die Biopsieplanung. Für die diffusionsgewichtete Bildgebung sollten hohe B-Werte angewandt werden. Die Fokussierung auf sogenannte Kurzprotokolle scheint dieselbe Aussagekraft hinsichtlich Detektion von Prostatakarzinomen zu haben - und dies bei einer Halbierung der Untersuchungszeit. Für das multiparametrische Prostata-MRT sind weiterhin falsch negative Befunde eine Herausforderung. Auch hinsichtlich der Nebennierendiagnostik ergeben sich Neuerungen durch die Verwendung von Dual-Energy-CT-Techniken. Ein Update zum Mammakarzinom gab Professor Wiesinger aus Tübingen. Insbesondere der moderne Mamma-Ultraschall inklusive Elastografie mittels qualitativer wie quantitativer Analyse, wie die kontrastverstärkte Mammografie, ist ein Ansatz, der sowohl Detektion als auch Grading deutlich verbessert hat. Die qualitative share-wave-Elastografie ist ein Prädiktor des histopathologi- schen Schweregrades. Insbesondere bei dichtem Brustdrüsenparenchym erhöht die kontrastverstärkte Mammografie die Detektion. Inwieweit Radiomics zu einer weiteren Verbesserung der Diagnostik führen ist noch offen.

Professor Spira aus Heidelberg fasste dann die Neuerungen bezüglich der Lungendiagnostik zusammen. Neben Änderungen im Lungenkrebsmanagement stellen neue Formen thorakaler Komplikationen durch molekulare Krebstherapien die Ärzte vor besondere diagnostische Herausforderungen. Auch hinsichtlich interstitiellen Pneumonien haben sich neue Aspekte ergeben. Technische Verbesserungen in der CT-Diagnostik und die Tomosynthese erhöhen die Sensitivität wie Spezifität der Thoraxdiagnostik weiter. Dr. Wolfang Fischer fasste dann die Neuerungen hinsichtlich der muskuloskelettalen Diagnostik zusammen. Neben technischen Aspekten standen neue Ansätze wie das compressed-sensing im Vordergrund, um eine schnelle Knorpelund Meniskusdarstellung zu ermöglichen. Die Quantifizierung des Fettgehalts der Wirbelsäule erlaubt eine bessere Einschätzung von Frakturen wie einer metastatischen Durchsetzung. Die Sarkopenie stellt einen interessanten neuen Aspekt bezüglich Frakturrisiko wie auch möglicher Tumorkomplikation dar. Die Sehnenqualität ist ein wichtiger Parameter in der Darstellung des Schultergelenks. Ramp-lesions standen im Fokus des Parts Kniebildgebung.

Im letzten Abschnitt stellte PD Dr. Radbruch aus Heidelberg/Essen die aktuelle Studienlage zu MR-Kontrastmitteln dar. Dies geschah in sorgfältiger Abwägung und Hinzuziehung pathophysiologischer Modelle. Eine besondere Bedeutung hat die Frage möglicher klinischer Korrelate. Der Unterschied zwischen den Empfehlun- gen der US-amerikanischen FDA vs. der europäischen EMA-Behörde wurde herausgearbeitet und zukünftige Entwicklungen dargestellt. Die aktuelle Studienlage Kinderradiologie wurde von Prof. Beer im letzten Vortrag zusammengefasst. Für angeborene wie erworbene Erkrankungen des ZNS und deren Therapie hat sich nochmals die Bedeutung der radiologischen Diagnostik wie der minimal-invasiven Therapie erhöht. Bezüglich Thorax- wie Abdomenbildgebung ergibt sich weiterhin ein Abwägen zwischen der Anwendung ionisierender Strahlung bei Röntgen- wie CT-Untersuchung und des diagnostischen Gewinns. Es zeigt sich insgesamt ein Shift zu strahlenfreien Verfahren wie Ultraschall und MRT. Hinsichtlich traumatischer Veränderungen bei Kindern ergibt sich eine zunehmende Anzahl von Sportverletzungen mit eigenen Verletzungsmustern. Auch entzündliche Erkrankungen des Knochens und der Muskulatur, wie die Osteomyelitis, die bakterielle Arthritis und die juvenile idiopathische Arthritis, zeigen eine Zunahme. Das MRT mit Diffusionswichtung erlaubt zunehmend eine exzellente Darstellung von Entzündungsprozessen.

Das spannende Format des RadiologieUpdates wird auch 2019 im März in Ulm fortgeführt werden.

Wir laden Sie herzlich vom 22.03. bis 23.03.2019 nach Ulm/Donau, CongressCentrum Ulm (CCU), ein.

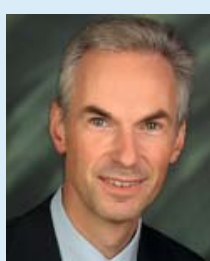

Prof. Dr. med. Meinrad Beer, Ulm 